\title{
Spontaneous motion of droplets during the demixing transition in binary fluids
}

\author{
V. Kumaran \\ Department of Chemical Engineering, Indian Institute of Science, Bangalore 560 012, India
}

\begin{abstract}
The convective interaction between a pair of droplets coarsening during the demixing transition of a binary fluid is examined. The starting point is the model $H$ equation for binary fluids, and the droplet sizes are considered to be large enough that thermal fluctuations are neglected. Droplet motion is induced by the convective coupling in the concentration equation, where there is a flux of concentration due to the fluid velocity, and a reciprocal effect in the momentum equation. The effect of the convective force density is separated into two parts-one due to the sharp concentration gradients at the droplet interface, and the other due to the variation in the matrix. It is shown that the dominant contribution to the fluid velocity field is due to the sharp concentration variation at the interface, and this is proportional to the square of the droplet flux at the surface. The surface flux is determined by solving the diffusion equation in the matrix between the droplets, and matching the solution to that in the interfacial region. The analysis indicates that there is an attractive interaction if the two droplets have radii larger or smaller than the critical radius, while the interaction is repulsive if the radius of one droplet is larger and the other smaller than the critical radius. The magnitude of the induced droplet velocity is estimated.
\end{abstract}

\section{INTRODUCTION}

There are two different types of morphologies that could develop during the late stages of the demixing transition in binary fluids. In a nearly symmetric quench, demixing proceeds due to the formation and coarsening of random interfaces which separate the two phases. In an asymmetric quench, droplets of the minority phase nucleate in the majority phase, and then grow due to diffusive transport or Brownian motion. The evolution of the morphology is very different in the two cases; the characteristic length scale of a random interface increases proportional to $t^{-1}$, while the characteristic droplet radius during droplet growth increases proportional to $t^{-1 / 3}$ in the case of diffusive growth as well as Brownian motion, where $t$ is the time after the quench. There have been many theoretical studies ${ }^{1-7}$ and computer simulations ${ }^{8,9}$ which have shown that there is a qualitative difference in the droplet growth process between metal alloys and fluid mixtures. This difference is because droplet coarsening could be enhanced by convective transport of material, in addition to the usual diffusion process that is responsible for the transport of material in solid lattices..$^{10,11}$ Experimental studies ${ }^{12-15}$ have also shown that the growth process is very different from the diffusion controlled growth processes in metal alloys and magnetic systems.

In addition to diffusive transport and Brownian motion, another mechanism for the coalescence of droplets has been identified in recent experiments. ${ }^{12,13}$ In these experiments, it was observed that even when the droplets are sufficiently large that Brownian motion is negligible, coalescence takes place due to the spontaneous motion of droplets towards each other. It was suggested that this could be due to the force exerted by the convective term in the momentum con- servation equation, which is the reciprocal of the convective transport term in the mass conservation equation in the model $H^{16}$ equations for a binary fluid. Further, it was also suggested that this force is caused by the sharp gradient in the concentration at the droplet interface. An analysis of the motion of a droplet in a steady concentration gradient was carried out by Tanaka ${ }^{17}$ to illustrate the effect of concentration gradient on droplet motion. Simulations in a two dimensional system ${ }^{18}$ have also demonstrated the attractive interaction between growing droplets in a supersaturated matrix.

Though experimental systems involve a large number of droplets simultaneously interacting with each other, the basic physics of the interaction is better illustrated by first considering a pair of droplets growing in a supersaturated matrix. Moreover, the multipole expansion method used here for calculating the interactions can be easily extended to a system with many droplets. The interaction between a pair of droplets of equal radius was analyzed earlier, ${ }^{5}$ and it was found that there is an attractive interaction between the droplets due to convective effects. In the present case, the analysis is extended to droplets of different sizes, and the droplet deformation is also examined. The calculation of the flow induced due to concentration variations is carried out in two steps. In the first step, it is shown that the sharp concentration gradients at the interface provide the dominant contribution to be convective force density and the induced fluid velocity, and the fluid velocity field is related to the flux at the surface. In the second step, the diffusion equation for the concentration field around a pair of droplets is solved to obtain the surface flux, and the motion of the droplets. The principal result of this analysis is that there is spontaneous droplet motion due 
to a contribution to the surface force density when the surface flux is nonzero, and this contribution is large compared to surface tension effects under nonequilibrium conditions.

\section{ANALYSIS}

The system consists of two droplets $A$ and $B$ with radii $R_{A}$ and $R_{B}$ separated by a vector distance $L$ growing due to diffusion in a matrix with a concentration $c_{\infty}$ which is higher than the saturation concentration. The free energy of the system is assumed to be of the usual Cahn-Hilliard square gradient form with an additional contribution due to the kinetic energy of the fluid

$$
F[c]=\int d \mathbf{x}\left[f[c]+\frac{K}{2}\left(\partial_{i} c\right)^{2}+\frac{\rho}{2} v_{i}^{2}\right],
$$

where indicial notation has been used for the vectors, $\partial_{i}$ $\equiv\left(\partial / \partial x_{i}\right), \rho$ is the fluid density and $v_{i}$ is the velocity. It is not necessary to specify $f[c]$ because, as discussed later, the motion of the droplets depends only on the square gradient term in the free energy. The conventional model $H$ equations ${ }^{16}$ are used for the concentration and velocity field in the fluid

$$
\begin{aligned}
& \partial_{t} c=\Lambda \partial_{i}^{2} \frac{\delta F}{\delta c}-v_{i} \partial_{i} c+\xi \\
& =\Lambda \partial_{i}^{2}\left[-K \partial_{j}^{2} c+\frac{\delta f[c]}{\delta c}\right]-v_{i} \partial_{i} c+\xi, \\
& \rho \partial_{t} v_{i}=\mu \partial_{j}^{2} v_{i}+\partial_{i} c \frac{\delta F}{\delta c}+\zeta_{i},
\end{aligned}
$$

where $\mu$ is the fluid viscosity, and $\xi$ and $\zeta_{i}$ are the appropriate noise sources required to satisfy the fluctuation dissipation theorem. The convective force density, which is the second term on the right side of (3), is reciprocal to the convective term in the mass conservation Eq. (2), and these two terms are required to ensure that the Poisson bracket relations are satisfied. The Langevin noise sources are neglected in the late stages of spinodal decomposition when the Brownian motion is negligible. In the concentration equation, the convective transport is considered to be small compared to the diffusion of solute, and the conditions for this assumption to be valid are examined a little later. With this simplification, the concentration equation is

$$
\partial_{t} c=\Lambda \partial_{i}^{2} \frac{\delta F}{\delta c} .
$$

The momentum equation is further simplified by neglecting fluid inertia, which is small in realistic systems ${ }^{5}$ to give

$$
\mu \partial_{j}^{2} v_{i}+\left[\partial_{i} c \frac{\delta F}{\delta c}\right]_{\perp}=0
$$

where $[\cdots]_{\perp}$ represents the transverse component. The above equation can be easily solved to give

$$
v_{i}(\mathbf{x})=\int d \mathbf{x}^{\prime} J_{i j}\left(\mathbf{x}-\mathbf{x}^{\prime}\right)\left[\partial_{j} c\left(\mathbf{x}^{\prime}\right) \frac{\delta F}{\delta c\left(\mathbf{x}^{\prime}\right)}\right]_{\perp},
$$

where the Oseen tensor $J_{i j}$ is

$$
J_{i j}(\mathbf{x})=\frac{1}{8 \pi \mu}\left[\frac{\delta_{i j}}{|\mathbf{x}|}+\frac{x_{i} x_{j}}{|\mathbf{x}|^{3}}\right] .
$$

A further simplification is possible in the expression for the velocity (5) by realizing that the convective force density due to the contribution proportional to $f[c]$ in the expression of the free energy is longitudinal

$$
\partial_{i} c \frac{\delta f[c]}{\delta c}=\partial_{i} f[c]
$$

and therefore does not contribute to the transverse part of the velocity field. The equation for the velocity in the fluid is then given by

$$
v_{i}(\mathbf{x})=-K \int d \mathbf{x}^{\prime} J_{i j}\left(\mathbf{x}-\mathbf{x}^{\prime}\right) \partial_{j} c\left(\mathbf{x}^{\prime}\right) \partial_{k}^{2} c\left(\mathbf{x}^{\prime}\right) .
$$

There are two length scales of interest in the problem, the magnitude of the droplet radius $R$ and the interfacial thickness $h$. The fluid velocity field can be separated into two contributions-one due to the sharp gradients in the concentration field at the droplet surface and the second due to the variation in the concentration in the matrix between the two droplets. It can be shown as follows that the velocity due to the nonequilibrium correction to the concentration field at the interface is large compared to that due to the variation in the matrix. For this purpose, it is useful to consider a specific model for the free energy $f[c]$,

$$
f[c]=\frac{\chi}{2}\left(c-c_{d}\right)^{2}\left(c-c_{m}\right)^{2}
$$

where $\chi$ is a constant, and $c_{d}$ and $c_{m}$ are the equilibrium droplet and matrix concentrations, respectively. The flux of material from the matrix to the droplet causes interface motion, and the concentration in the reference frame fixed on the interface is determined from the concentration equation

$$
\begin{aligned}
-u\left(\mathbf{x}_{s}\right) \partial_{z} c= & \Lambda \partial_{z}^{2}\left[\frac{\delta F}{\delta c}\right] \\
= & \Lambda \partial_{z}^{2}\left[-K \partial_{z}^{2} c+2 \chi\left(c-c_{d}\right)\right. \\
& \left.\times\left(c-c_{m}\right)\left(2 c-c_{d}-c_{m}\right)\right],
\end{aligned}
$$

where $z$ is the coordinate normal to the interface, and $u\left(\mathbf{x}_{s}\right)$ is the normal velocity of the interface due to the flux of solute. The equilibrium concentration profile is

$$
c_{e}=\frac{c_{d}+c_{m}}{2}-\frac{c_{d}-c_{m}}{2} \tanh \left(\frac{z}{h}\right),
$$

where $h=\left[2 K / \chi\left(c_{d}-c_{m}\right)^{2}\right]^{1 / 2}$ is the interfacial thickness. In the above expression, curvature affects have been neglected since the interface thickness is small compared to the radius of the droplet. It is useful to express the concentration profile as $c=\left(c_{d}+c_{m}\right) / 2+\psi\left(c_{d}-c_{m}\right) / 2$. The equilibrium interface concentration is then

$$
\psi_{e}=-\tanh \left(\frac{z}{h}\right)
$$




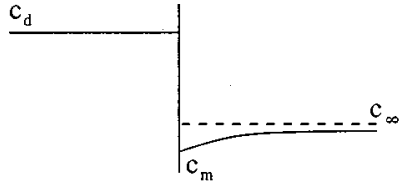

(a)

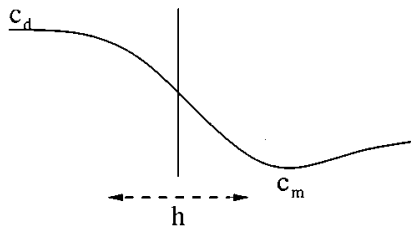

(b)
FIG. 1. Schematic of the variation of the concentration field on the scale of the droplet radius (a) and on the scale of the interfacial thickness (b).

The nonequilibrium correction to the interfacial concentration profile is determined by linearizing the concentration equation in the departure from equilibrium $\phi=\psi-\psi_{e}$,

$$
-\frac{u\left(\mathbf{x}_{s}\right)}{h} \partial_{z *} \psi_{e}=\frac{D}{h^{2}} \partial_{z *}^{2}\left[-\frac{1}{2} \partial_{z *}^{2} \phi+\left(\frac{3 \psi_{e}^{2}-1}{2}\right) \phi\right],
$$

where the dimensionless coordinate $z^{*}=(z / h)$, and $D$ $=\Lambda_{\chi}\left(c_{d}-c_{m}\right)^{2}$ is the bulk diffusion coefficient. The above fourth order equation can be integrated twice to obtain

$$
\begin{aligned}
& {\left[-\frac{1}{2} \partial_{z^{*}}^{2} \phi+\left(\frac{3 \psi_{e}^{2}-1}{2}\right) \phi\right]} \\
& \quad=\frac{u h}{D}\left[\int_{0}^{z^{*}} d y\left(-\psi_{e}(y)\right)+z^{*}-\log (2)\right],
\end{aligned}
$$

where the constants of integration are determined from the requirement that the $\phi=0$ and $d_{z *} \phi=0$ in the droplet phase $z^{*} \rightarrow-\infty$, where the concentration is equal to the equilibrium concentration. It is not necessary to obtain the numerical solution to Eq. (14) for $\phi$, but is sufficient to recognize the following limiting behavior of the function $\phi\left(z^{*}\right)$,

$$
\begin{aligned}
& \phi \propto \exp \left(2 z^{*}\right) \quad \text { for } z^{*} \rightarrow-\infty, \\
& \phi=\frac{2 u\left(\mathbf{x}_{s}\right) h}{D} z^{*} \text { for } z^{*} \rightarrow \infty .
\end{aligned}
$$

The above limiting behavior is accurate only for $z \sim h$ at the interface, and this is not applicable for $z \sim R$ because there is a correction due to curvature effects. A schematic of the variation in the concentration field on the scale of the droplet radius is shown in Fig. 1(a), and on the scale of the interface thickness $h$ is shown in Fig. 1(b).

The concentration field in the matrix is determined by solving the diffusion equation (4). It can be shown, as follows, that this reduces to the Laplace equation for the concentration field in the matrix. The concentration field in the matrix is expressed as $c=c_{\infty}+c^{*}$, where $c^{*}$ is the difference between the local concentration and the background concentration $c_{\infty}$ at a large distance from the droplet. The concentration equation (4) is expressed in terms of $c^{*}$, and linearized in the limit $\left(c_{\infty}-c_{m}\right) \ll\left(c_{d}-c_{m}\right)$ appropriate for late stage growth, to obtain

$$
\partial_{t} c^{*}=\Lambda \partial_{i}^{2}\left[-K \partial_{j}^{2} c^{*}+\chi\left(c_{d}-c_{m}\right)^{2} c^{*}\right] .
$$

In the above equation, it can easily be verified that the first term on the right is $O\left(h^{2} / R^{2}\right)$ smaller than the second, since the length scale for the variation of $c^{*}$ is $R$. With this simplification, the concentration equation reduces to

$$
\partial_{t} c^{*}=D \partial_{j}^{2} c^{*}
$$

where the diffusion coefficient $D=\Lambda \chi\left(c_{d}-c_{m}\right)^{2}$. If the motion of the interface is due to diffusion, the left-hand side of the above equation can be estimated as $\left(u c^{*} / R\right)$, where $u$, the normal velocity of the interface, is $O\left(D c^{*} / R\left(c_{d}-c_{m}\right)\right)$. It is easily seen that in the late stage when the supersaturation is small and $c^{*} \ll\left(c_{d}-c_{m}\right)$, the left-hand side of (18) is small compared to the right-hand side. However, there is droplet motion due to convective effects as well, and it is shown a little later that the velocity field in this case scales as $\left(K\left(c_{\infty}-c_{m}\right)^{2} R / L^{2} \mu\right)$, where $\mu$ is the fluid viscosity and $L$ is the distance between the interacting droplets. It can easily be verified from (1) that the surface tension $\sigma \sim K\left(c_{d}-c_{m}\right)^{2} / h$, and therefore the fluid velocity due to convective effects scales as $\left(\sigma\left(c_{\infty}-c_{m}\right)^{2} R h / L^{2}\left(c_{d}-c_{m}\right)^{2} \eta\right)$. This velocity could be significant even though the ratio $\left(c_{\infty}-c_{m}\right) /\left(c_{d}\right.$ $\left.-c_{m}\right)$ and $(h / L)$ are small, because the ratio $(\sigma / \eta)$ could be quite large. For example, the surface tension and viscosity for water are $O\left(10^{-2} \mathrm{~kg} / \mathrm{s}^{2}\right)$ and $10^{-3} \mathrm{~kg} / \mathrm{m} / \mathrm{s}$, respectively, and so the ratio $(\sigma / \eta)$ is $O(10 \mathrm{~m} / \mathrm{s})$. Therefore, even if the ratio $\left(R h / L^{2}\right)\left(c_{\infty}-c_{m}\right)^{2} /\left(c_{d}-c_{m}\right)^{2}$ is $O\left(10^{-4}\right)$, the convective velocity is still of $O(1 \mathrm{~mm} / \mathrm{s})$. The ratio of the convective and diffusive terms in the conservation equation is $O\left(\sigma h R^{2} / L^{2} \eta D\right)\left(c_{\infty}-c_{m}\right)^{2} /\left(c_{d}-c_{m}\right)^{2}$. In the present analysis, this parameter is considered to be small, and diffusion is considered to be dominant in the leading approximation. In the absence of convection, the concentration equation reduces to

$$
\partial_{j}^{2} c^{*}=0 .
$$

It can also be inferred that the convective force density in the matrix, which is proportional to $\partial_{j}^{2} c$, is also zero in the leading approximation. Consequently, it is sufficient to consider the fluid flow due to the convective force density at the interface alone.

For sharp interfaces, the integral in (9) can be separated into the product of integrals over the coordinate normal to the interface $z$ and the area of the droplet $A$ :

$v_{i}(\mathbf{x})=-K \int d A J_{i j}\left(\mathbf{x}-\mathbf{x}_{s}\right) \int d z \partial_{i} c\left(\mathbf{x}^{\prime}\right) \partial_{j}^{2} c\left(\mathbf{x}^{\prime}\right)$,

where $\mathbf{x}_{s}$ is the position along the surface of the droplet. In deriving the above equation, the Oseen tensor $J_{i j}\left(\mathbf{x}-\mathbf{x}^{\prime}\right)$ $=J_{i j}\left(\mathbf{x}-\mathbf{x}_{s}-z \mathbf{n}\right)$ has been approximated by $J_{i j}\left(\mathbf{x}-\mathbf{x}_{s}\right)$. The error due to this approximation is $O(h / R)$, since the concentration gradients are significant only over the interfacial region of thickness $h$. At equilibrium, it can be shown that the term $-K \int d z \partial_{i} c\left(\mathbf{x}^{\prime}\right) \partial_{j}^{2} c\left(\mathbf{x}^{\prime}\right)$ gives the normal force exerted by the interface, which is the product of the surface tension, mean curvature and the unit normal. The dominant contribution to the term $\partial_{i} c$ at equilibrium is $n_{i} \partial_{z} c$, since there are no concentration variations in the tangential direction. The term $\partial_{j}^{2} c$ can be written as

$$
\partial_{j}^{2} c=\partial_{z}^{2} c+\left(\frac{1}{R_{1}}+\frac{1}{R_{2}}\right) \partial_{z} c+O\left(\frac{h^{2}}{R^{2}}\right),
$$


where $R_{1}$ and $R_{2}$ are the principal radii of curvature at a point on the interface, and the second term on the right-hand side of (21) is because the coordinate $z$ is measured from a curved surface. Using the above relations, the term

$$
\begin{aligned}
& -K \int d z \partial_{i} z \partial_{j}^{2} z \\
& \quad=-K n_{i} \int d z\left[\partial_{z} c \partial_{z}^{2} c+\left(\frac{1}{R_{1}}+\frac{1}{R_{2}}\right)\left(\partial_{z} c\right)^{2}\right] \\
& \quad=-K n_{i}\left[\left.\left(\partial_{z} c\right)^{2}\right|_{-\infty} ^{\infty}+\left(\frac{1}{R_{1}}+\frac{1}{R_{2}}\right) \int d z\left(\partial_{z} c\right)^{2}\right] .
\end{aligned}
$$

At equilibrium, it is easily verified that the first term on the right-hand side of (22) is identically zero, because $\partial_{z} c$, which is the flux of solute at the interface, is zero. The second term on the right-hand side of (22) is the product of the unit normal $\left(n_{i}\right)$, the curvature $\left[\left(1 / R_{1}\right)+\left(1 / R_{2}\right)\right]$, and the surface tension $K \int d z\left(\partial_{z} c\right)^{2}$; and the negative sign in (22) indicates that the force due to surface tension is directed opposite to the outward unit normal $n_{i}$. In a nonequilibrium system, the flux at the interface is nonzero, and consequently the first term on the right-hand side of (22) is nonzero. Moreover, this term is $O(R / h)$ larger than the second term, and consequently provides the dominant contribution to the convective force density at the interface, and the second term on the right-hand side can be neglected in comparison to the first. With this approximation, the velocity field is

$$
\begin{aligned}
v_{i}(\mathbf{x})= & -K \int d A J_{i j}\left(\mathbf{x}-\mathbf{x}_{s}\right) n_{j}\left(\mathbf{x}_{s}\right) \\
& \times \int d z \partial_{z} c\left(\mathbf{x}_{s}+\mathbf{n} z\right) \partial_{z}^{2} c\left(\mathbf{x}_{s}+\mathbf{n} z\right) \\
= & -K \int d A J_{i j}\left(\mathbf{x}-\mathbf{x}_{s}\right) n_{j}\left(\mathbf{x}_{s}\right)\left[\frac{\left(\partial_{z} c\left(\mathbf{x}_{s}+\mathbf{n} z\right)\right)^{2}}{2}\right]_{-\infty}^{\infty} .
\end{aligned}
$$

Since the droplet concentration has attained its equilibrium value, $\partial_{z} c=0$ in the limit $z \rightarrow-\infty$. The gradient of the interfacial concentration profile in the region $z \rightarrow \infty$ is determined by matching the interfacial solution valid for $z \sim h$ and the outer solution in the matrix valid for $z \sim R$ in the intermediate region $z \gg h$ and $z \ll R$. It is shown a little later that this matching condition reduces to the requirement that the concentration at the interface obtained from the outer solution should be equal to the equilibrium matrix concentration, and the concentration gradient $\partial_{z} c=\left(j_{n} / D\right)$, where $j_{n}$ is the normal flux at the interface obtained from the outer solution. With these simplifications, the expression for the fluid velocity reduces to

$$
v_{i}(\mathbf{x})=-\frac{K}{2 D^{2}} \int d A J_{i j}\left(\mathbf{x}-\mathbf{x}_{s}\right)\left[j_{n}\left(\mathbf{x}_{s}\right)\right]^{2} .
$$

It can be seen from (24) that the fluid velocity field depends on the flux of solute at the interface of the droplet. The concentration field and the interfacial flux due to the interaction between a pair of droplets is examined next. The boundary conditions at the droplet surfaces are determined by matching the inner solution in the interfacial region (16) with the outer solution obtained by solving (19) in the intermediate region $h \ll z \ll R$. In this region, the inner solution for the concentration field scales as $c_{m}+O\left(u z\left(c_{d}-c_{m}\right) / D\right)$ from (16), which is $c_{m}+O\left(O\left(z\left(c_{\infty}-c_{m}\right) / R\right)\right)$ since the velocity of the interface $u$ is $O\left((D / R)\left(c_{\infty}-c_{m}\right) /\left(c_{d}-c_{m}\right)\right)$. Thus, the deviation of the concentration field from its equilibrium value $c_{m}$ in the matrix is $O\left(z\left(c_{\infty}-c_{m}\right) / R\right)$, which is small compared to the magnitude of the concentration variation in the outer region $\left(c_{\infty}-c_{m}\right)$ in the limit $h \ll z \ll R$. Consequently, it is appropriate to set the concentration at the interface equal to the equilibrium concentration $c_{m}$ while obtaining the outer solution.

The radius of the droplet in the absence of Brownian motion or convection is determined by the Lifshitz-Slyozov theory, ${ }^{11}$ where the droplet concentration is assumed to have reached its equilibrium value, but the matrix is supersaturated. The chemical potential for the droplet phase contains an additional contribution due to the Laplace pressure arising from the surface tension between the droplet and matrix, while the chemical potential of the matrix is higher than the equilibrium chemical potential. When the droplet size is smaller than a critical value, the chemical potential of the droplet (which contains a contribution proportional to the inverse of the radius due to the Laplace pressure) is higher than that of the matrix, and the droplet shrinks. When the droplet size is larger than the critical value, the chemical potential of the droplet phase is lower than that of the supersaturated matrix and the droplet grows. The critical radius (for a given supersaturation of the matrix) is the radius at which the droplet neither shrinks nor grows. The equilibrium concentration of the matrix at the droplet surface depends on the radius of the droplet, and the equilibrium concentrations for two interacting droplets with radius $R_{A}$ and $R_{B}$ are ${ }^{11}$

$$
c_{A}=C_{e}+\frac{C^{\prime}}{R_{A}}, \quad c_{B}=C_{e}+\frac{C^{\prime}}{R_{B}},
$$

where $C_{e}$ is the equilibrium concentration above a plane surface, and $\left(C^{\prime} / R_{A}\right)$ and $\left(C^{\prime} / R_{B}\right)$ are the corrections to the equilibrium concentration due to the curvature of the droplet. It is now necessary to solve the Laplace equation for the concentration field (19) with the boundary conditions $c^{*}$ $=-c_{A}^{*}=c_{\infty}-c_{A}$ at the surface of droplet $A$ and $c^{*}=-c_{B}^{*}$ $=c_{\infty}-c_{B}$ at the surface of droplet $B$. The critical radius ${ }^{11}$ is given by $R_{c}=C^{\prime} /\left(c_{\infty}-C_{e}\right)$, and $c^{*}=0$ at the interface for droplets with the critical radius. In the theory for late stage droplet growth, droplets with radius larger than the critical radius grow due to diffusion of solute into the droplet, while droplets with radius smaller than the critical radius shrink due to diffusion from the droplet to the matrix.

The solution is obtained using multipole expansions, where the concentration field is determined as an expansion in spherical harmonics in two coordinate systems with origins at the centers of the two droplets. The solution for the concentration field is obtained as an asymptotic expansion in the parameters $\left(R_{A} / L\right)$ and $\left(R_{B} / L\right)$, where $L$ is the distance between the two droplets. Two spherical coordinate systems $\left(r_{A}, \theta_{A}, \phi_{A}\right)$ and $\left(r_{B}, \theta_{B}, \phi_{B}\right)$ with origins at the centers of the two droplets are chosen as shown in Fig. 2. The concentra- 


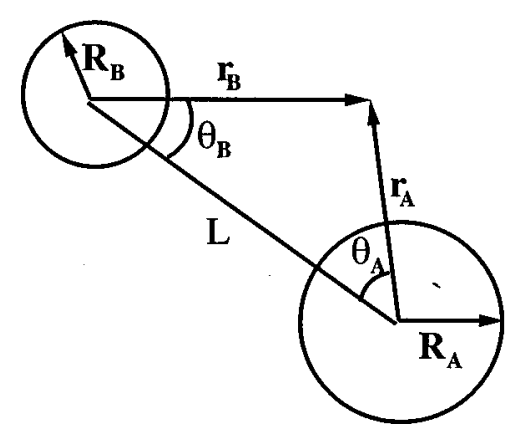

FIG. 2. Coordinate systems used for calculating the concentration field around a pair of droplets.

tion field is axisymmetric about the line joining the centers of the two droplets, and the solution for the concentration field is given by

$c^{*}=\sum_{n=0}^{\infty}\left(\frac{A_{n}}{r_{A}^{n+1}} P_{n}\left(\cos \left(\theta_{A}\right)\right)+\frac{B_{n}}{r_{B}^{n+1}} P_{n}\left(\cos \left(\theta_{B}\right)\right)\right)$,

where $P_{n}(x)$ are Legendre polynomials. The coefficients $A_{n}$ and $B_{n}$ are expressed as an asymptotic expansion in the parameters $\left(R_{A} / L\right)$ and $\left(R_{B} / L\right)$,

$$
\begin{aligned}
& A_{n}=\sum_{m=0}^{\infty} A_{n m}\left(\frac{R_{A}}{L}\right)^{m}, \\
& B_{n}=\sum_{m=0}^{\infty} B_{n m}\left(\frac{R_{B}}{L}\right)^{m}
\end{aligned}
$$

and the coefficients $A_{n m}$ and $B_{n m}$ are chosen to satisfy the boundary conditions (25) at the surfaces of the two droplets. For this purpose, it is necessary to express the spherical harmonics in the coordinate system centered at droplet $A$ in terms of those centered at droplet $B$ and vice versa. These relations are provided by Hobson ${ }^{19}$

$$
\begin{aligned}
& \left(\frac{1}{r_{A}}\right)^{n+1} P_{n}\left(\cos \left(\theta_{A}\right)\right) \\
& =\left(\frac{1}{L}\right)^{n+1} \sum_{q=0}^{\infty}\left(\begin{array}{c}
n+q \\
q
\end{array}\right)\left(\frac{r_{B}}{L}\right)^{q} P_{q}\left(\cos \left(\theta_{B}\right)\right), \\
& \left(\frac{1}{r_{B}}\right)^{n+1} P_{n}\left(\cos \left(\theta_{B}\right)\right) \\
& =\left(\frac{1}{L}\right)^{n+1} \sum_{q=0}^{\infty}\left(\begin{array}{c}
n+q \\
q
\end{array}\right)\left(\frac{r_{A}}{L}\right)^{q} P_{q}\left(\cos \left(\theta_{A}\right)\right)
\end{aligned}
$$

for $r_{A}<L$ and $r_{B}<L$. Using the above relations, the coefficients $A_{n m}$ and $B_{n m}$ are

$$
\begin{aligned}
A_{0 m}= & -c_{A}^{*} \delta_{0 m}, \\
A_{n m}= & -\left(\frac{R_{A}}{R_{B}}\right)^{n-m[}\left[\sum_{q=0}^{m-n-1} B_{q(m-n-q-1)}\left(\begin{array}{c}
n+q \\
q
\end{array}\right)\right] \\
& \text { for } n>0, \\
B_{0 m}= & -c_{B}^{*} \delta_{0 m}, \\
B_{n m}= & -\left(\frac{R_{B}}{R_{A}}\right)^{n-m}\left[\sum_{q=0}^{m-n-1} A_{q(m-n-q-1)}\left(\begin{array}{c}
n+q \\
q
\end{array}\right)\right]
\end{aligned}
$$

for $n>0$.

The normal flux at the surfaces of $\operatorname{droplet} A$ and $B$ are then obtained from the above solution

$$
\begin{aligned}
j_{A}= & -\left.D \partial_{r A} c^{*}\right|_{r_{A}=R_{A}} \\
= & -D \sum_{n=0}^{\infty} \sum_{m=0}^{\infty}\left[\left(-\frac{(n+1) A_{n m}}{R_{A}}\right)\left(\frac{R_{A}}{L}\right)^{m}+\sum_{q=0}^{m-n-1}\left(\frac{n B_{q m}}{R_{A}}\right)\right. \\
& \left.\times\left(\frac{R_{B}^{m+q+1} R_{A}^{n}}{L^{n+m+q+1}}\right)\left(\begin{array}{c}
n+q \\
n
\end{array}\right)\right] P_{n}\left(\cos \left(\theta_{A}\right)\right), \\
j_{B}= & -\left.D \partial_{r B} c^{*}\right|_{r_{B}=R_{B}} \\
= & -D \sum_{n=0}^{\infty} \sum_{m=0}^{\infty}\left[\left(-\frac{(n+1) B_{n m}}{R_{B}}\right)\left(\frac{R_{B}}{L}\right)^{m}\right. \\
& \left.+\sum_{q=0}^{m-n-1}\left(\frac{n A_{q m}}{R_{B}}\right)\left(\frac{R_{A}^{m+q+1} R_{B}^{n}}{L^{n+m+q+1}}\right)\left(\begin{array}{c}
n+q \\
q
\end{array}\right)\right] P_{n}\left(\cos \left(\theta_{B}\right)\right) .
\end{aligned}
$$

The fluid velocity field can now be calculated using Eq. (24), and the velocity of the surface of the droplet thereby evaluated. It is convenient to determine the radial velocity of the droplet surface using an expansion in Legendre polynomials

$$
v_{A r}\left(\theta_{I}\right)=\sum_{i=n}^{\infty} V_{A}^{(n)} P_{n}\left(\cos \left(\theta_{A}\right)\right)
$$

The radial velocity does not contain a component proportional to $P_{0}\left(\cos \left(\theta_{A}\right)\right)$ due to the incompressibility requirement. The coefficients $V_{A r}^{(n)}$ are determined using Eq. (24) and the orthogonality condition for Legendre polynomials

$$
\begin{aligned}
V_{A}^{(n)}= & -\frac{K}{2 D^{2}} \sum_{J=A, B} \int d \mathbf{x}_{J S}^{\prime} n_{j}\left(\mathbf{x}_{J S}^{\prime}\right)\left[j\left(\mathbf{x}_{J S}^{\prime}\right)\right]^{2} \\
& \int d \mathbf{x}_{A s} n_{i}\left(\mathbf{x}_{A s}\right) J_{i j}\left(\mathbf{x}_{A s}-\mathbf{x}_{J S}^{\prime}\right) \frac{(2 n+1)}{4 \pi R_{A}^{2}} P_{n}\left(\cos \left(\theta_{A}\right)\right) .
\end{aligned}
$$

The coefficient $V_{A r}^{(1)}$ provides the translational velocity of the droplet, while the higher order terms give the rate of deformation. The details of the calculation of the integrals in (33) are provided in the Appendix, and the results for $V_{A}^{(n)}$ for 0 $\leqslant n \leqslant 5$ correct to $O\left(1 / L^{5}\right)$ are also listed in the Appendix.

Due to symmetry considerations, the droplets are constrained to move along the line joining their centers, and the 
velocity perpendicular to the line joining their centers is zero. The translational velocity of the droplet along the line joining the centers is determined as the average of the velocity over along the line joining the centers over the surface of the droplet:

$$
V_{A}=\frac{1}{S} \int d S \cos \left(\theta_{A}\right) v_{A r},
$$

where $S$ is the surface of the droplet, and the factor $\cos \left(\theta_{A}\right)$ provides the component of the radial along the line joining the centers. It can be easily verified that when the velocity is expanded in a Legendre polynomial series (32), the integral (34) provides

$$
V_{A}=V_{A}^{(1)} \text {. }
$$

It is useful to examine the motion and deformation of the droplets when the distance of separation is large compared to the droplet radius. The leading order contribution to the mean velocity of the droplet, $V_{A}^{(1)}$, proportional to $\left(1 / L^{2}\right)$, is

$$
V_{A}^{(1)}=\frac{4 K c_{A}^{*} c_{B}^{*} R_{B}}{5 L^{2}}+O\left(1 / L^{3}\right) .
$$

In the limit of large $L$, this velocity is positive if the product $\left(c_{A}^{*} c_{B}^{*}\right)$ is positive, and the droplets approach each other along their line of centers. Thus, two droplets with radii greater than or less than the critical radius approach each other, while a pair of droplets with one larger and the other smaller than the critical radius move away. This physical reason for this behavior is shown in Fig. 3. When the two droplets are either larger or smaller than the critical radius, the flux on the part of the droplet surface facing the other droplet is smaller than that on the part not facing the other droplet. Since the velocity at a point on the surface depends on the square of the normal flux at that point from (24), this variation of the flux results in the two droplets coming towards each other. When one of the droplets is larger and the other smaller than the critical radius, the flux in between the two droplets is larger than that on the parts of the surface not facing the other droplet. This results in the droplets moving away from each other.

The leading order contribution to the deformation rate $V_{A}^{(2)}$, proportional to $\left(1 / L^{3}\right)$, is

$$
V_{A}^{(2)}=\frac{4 K c_{A}^{*} c_{B}^{*} R_{A} R_{B}}{L^{3}}+O\left(1 / L^{4}\right) .
$$

This coefficient is also positive for $c_{A}^{*} c_{B}^{*}>0$, indicating that droplets which approach each other deform into prolate spheroids which are elongated along the line joining the centers, while the coefficient is negative for $c_{A}^{*} c_{B}^{*}<0$ indicating that droplets which move apart deform into oblate spheroids which are compressed along the line joining their centers.

\section{CONCLUSIONS}

The interaction between a pair of droplets in the demixing transition in a binary fluid has been examined in the late stages where the Brownian diffusion is not important. The model $H$ concentration and momentum equations were used as the starting point for the analysis, and inertial effects and

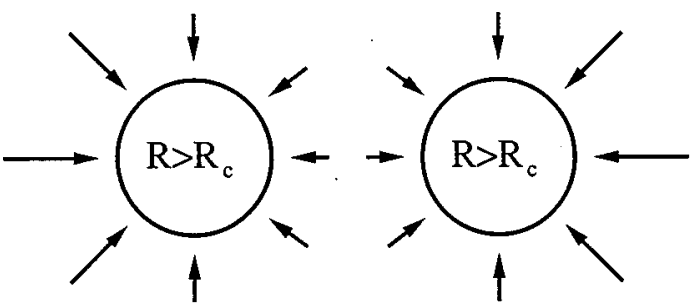

(a)

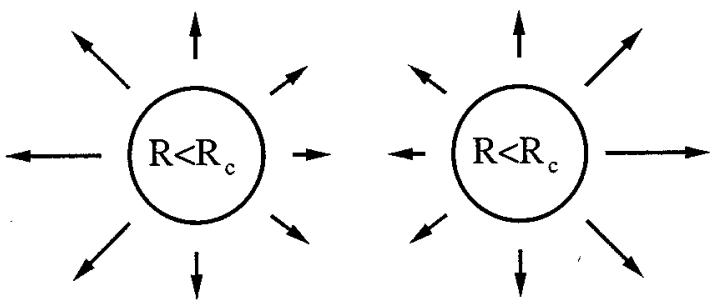

(b)

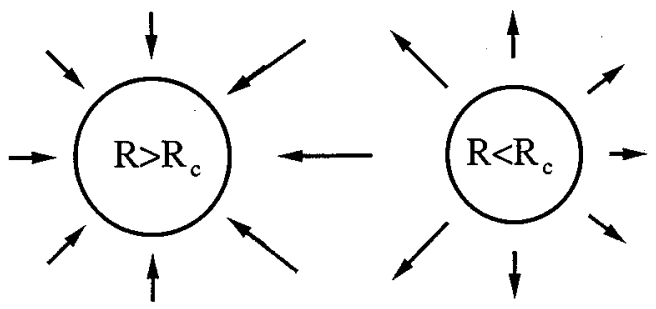

(c)

FIG. 3. Variation in the surface flux due to the interaction between two droplets (a) both of which have radii larger than the critical radius; (b) both of which have radii smaller than the critical radius; (c) one has radius larger and the other smaller than the critical radius.

Brownian motion were neglected in this analysis. The convective interaction arises due to the presence of the convective terms in the concentration equation, and the reciprocal term in the momentum equation required for satisfying the fluctuation dissipation theorem. The effect of the convective term in the momentum equation was separated into two parts - one due to the sharp concentration gradients at the interface and the other due to the variations in the matrix between two droplets. It was shown that the dominant effect is due to the sharp concentration variations at the interface under nonequilibrium conditions, and this effect was proportional to the square of the solute flux at the interface. The flux at the interface was determined by solving the diffusion equation in the matrix between the two droplets, and matching the solution with that in the interfacial region.

The results of the analysis indicate that convective effects do result in spontaneous motion of the droplets. If both droplets have radii that are larger or smaller than the critical radius, then the droplets approach each other, while if one has a larger radius and the other has a smaller radius, then there is a repulsive interaction. The physical reason for this is shown in Fig. 3. In addition, the deformation of the droplets could also be determined in the present analysis, and it was found that droplets attracted to each other deform into prolate spheroids along the line of centers, while droplets that are repelled deform into oblate spheroids.

It is useful to estimate the magnitude of the droplet velocities. It can easily be verified from (1) that the surface 
tension $\sigma \sim K\left(c_{d}-c_{m}\right)^{2} / h$, where $c_{d}$ is the droplet concentration and $c_{m}$ is the concentration in the matrix which is in equilibrium with the droplet phase. Therefore the fluid velocity due to convective effects scales as $\left(\sigma\left(c_{\infty}\right.\right.$ $\left.\left.-c_{m}\right)^{2} R h / L^{2}\left(c_{d}-c_{m}\right)^{2} \eta\right)$. The ratio $(R / L) \sim 1$, and $(h / L)$ $\sim 10^{-3}$ for typical values of $R \sim 1 \mu \mathrm{m}$ and $h \sim 1 \mathrm{~nm}$. The surface tension and viscosity for water are $O\left(10^{-2} \mathrm{~kg} / \mathrm{s}^{2}\right)$ and $10^{-3} \mathrm{~kg} / \mathrm{m} / \mathrm{s}$, respectively, and so the ratio $(\sigma / \eta)$ is $O(10 \mathrm{~m} / \mathrm{s})$. Therefore, even if the ratio $\left(c_{\infty}-c_{m}\right)^{2} /\left(c_{d}\right.$ $\left.-c_{m}\right)^{2}$ is in the range $10^{-2}-10^{-4}$, the velocity is $1-100 \mu \mathrm{m} / \mathrm{s}$, which could be significant.

\section{APPENDIX}

The calculation of the integrals in (33) is discussed in this section. The expression for $V_{A}^{(n)}$ can be separated into two parts, $V_{A A}^{(n)}$ and $V_{A B}^{(n)}$, which represent the Legendre modes of the velocity induced at the surface of droplet $A$ due to the flux at the surface of droplet $A$ and $B$, respectively,

$$
\begin{aligned}
V_{A A}^{(n)}= & -\frac{K}{2 D^{2}} \int d \mathbf{x}_{A s}^{\prime} n_{j}\left(\mathbf{x}_{A s}^{\prime}\right)\left[j\left(\mathbf{x}_{A s}^{\prime}\right)\right]^{2} \\
& \times \int d \mathbf{x}_{A s} n_{i}\left(\mathbf{x}_{A s}\right) J_{i j}\left(\mathbf{x}_{A s}-\mathbf{x}_{A s}^{\prime}\right) \frac{(2 n+1)}{4 \pi R_{A}^{2}} P_{n}\left(\cos \left(\theta_{A}\right)\right), \\
V_{A B}^{(n)}= & -\frac{K}{2 D^{2}} \int d \mathbf{x}_{B s}^{\prime} n_{j}\left(\mathbf{x}_{B s}^{\prime}\right)\left[j\left(\mathbf{x}_{B s}^{\prime}\right)\right]^{2} \int d \mathbf{x}_{A s} n_{i}\left(\mathbf{x}_{A s}\right) \\
& \times J_{i j}\left(\mathbf{x}_{A s}-\mathbf{x}_{A s}^{\prime}-\mathbf{L}\right) \frac{(2 n+1)}{4 \pi R_{A}^{2}} P_{n}\left(\cos \left(\theta_{A}\right)\right) .
\end{aligned}
$$

The first component $V_{A A}^{(n)}$ is determined as follows. The coordinate system shown in Fig. 2 is employed, where the azimuthal angle between $\mathbf{x}_{A s}^{\prime}$ and the line joining the centers of the two droplets is $\theta_{A}^{\prime}$, and the azimuthal and meridional angles between $\mathbf{x}_{A s}$ and $\mathbf{x}_{A s}^{\prime}$ are $\left(\theta^{*}, \phi^{*}\right)$, respectively. The azimuthal angle between the vector $\mathbf{x}_{A s}$ and the line joining the centers of the droplets is then given by

$$
\cos \left(\theta_{A}\right)=\cos \left(\theta^{*}\right) \cos \left(\theta_{A}^{\prime}\right)-\sin \left(\theta^{*}\right) \sin \left(\theta_{A}^{\prime}\right) \cos \left(\phi^{*}\right) .
$$

The function $n_{i}\left(\mathbf{x}_{A s}\right) J_{i j}\left(\mathbf{x}_{A s}-\mathbf{x}_{A s}^{\prime}\right) n_{j}\left(\mathbf{x}_{A s}^{\prime}\right)$ can be expressed in terms of the angles $\theta_{A}^{\prime}, \theta^{*}$, and $\phi^{*}$ :

$$
\begin{aligned}
& n\left(\mathbf{x}_{A s}\right) J_{i j}\left(\mathbf{x}_{A s}-\mathbf{x}_{A s}^{\prime}\right) n_{j}\left(\mathbf{x}_{A s}^{\prime}\right) \\
& =\frac{3 \cos \left(\theta^{*}\right)-1}{8 \pi \mu R_{A}(2 \sqrt{2})\left[1-\cos \left(\theta^{*}\right)\right]^{1 / 2}} .
\end{aligned}
$$

Using the above expressions, the component $V_{A A}^{(n)}$ is

$$
V_{A A}^{(n)}=\frac{1}{8 \pi \mu} \int d \mathbf{x}_{A s}^{\prime}\left[j\left(\mathbf{x}_{A s}^{*}\right)\right]^{2} h_{n} P_{n}\left(\cos \left(\theta_{A}^{\prime}\right)\right),
$$

where

$$
\begin{aligned}
h_{n}= & \frac{1}{P_{n}\left(\cos \left(\theta_{A}^{\prime}\right)\right)} \frac{2 n+1}{2} \int_{0}^{\pi} d \theta_{A} n_{i}\left(\mathbf{x}_{A s}\right) \\
& \times J_{i j}\left(\mathbf{x}_{A s}-\mathbf{x}_{A s}^{\prime}\right) n_{j}\left(\mathbf{x}_{A s}\right) P_{n}\left(\cos \left(\theta_{A}\right)\right) .
\end{aligned}
$$

Using (A3), the coefficients $h_{n}$ for $0 \leqslant n \leqslant 5$ are $h_{0}=0, h_{1}$ $=(8 / 5), \quad h_{2}=(8 / 7), \quad h_{3}=(16 / 15), \quad h_{4}=(80 / 7), \quad$ and $h_{5}$ $=(40 / 39)$, respectively.

The second component $V_{A B}^{(n)}$ is determined using an asymptotic expansion in the parameters $\left(R_{A} / L\right)$ and $\left(R_{B} / L\right)$

$$
\begin{aligned}
V_{A B}^{(n)}= & \frac{1}{8 \pi \mu} \int d \mathbf{x}_{B s}^{\prime} n_{j}\left(\mathbf{x}_{B s}^{\prime}\right) \\
& \times\left[\left(\mathbf{x}_{B s}^{\prime}\right)\right]^{2} \sum_{n} \sum_{m} \frac{g_{m n}}{L^{m}} P_{n}\left(\cos \left(\theta_{B}^{\prime}\right)\right),
\end{aligned}
$$

where the coefficients $g_{m n}$ determined as follows. The coordinate system shown in Fig. 2 is used for the analysis, and the product

$$
n_{i}\left(\mathbf{x}_{A s}\right) J_{i j}\left(\mathbf{x}_{A s}-\mathbf{x}_{B s}^{\prime}\right) n_{j}\left(\mathbf{x}_{B s}^{\prime}\right)=\left(\frac{\cos \left(\theta^{*}\right)}{r}+\frac{\left(R_{A}-R_{B} \cos \left(\theta^{*}\right)-L \cos \left(\theta_{A}\right)\right)\left(R_{A} \cos \left(\theta^{*}\right)-R_{B}+L \cos \left(\theta_{B}^{\prime}\right)\right)}{r^{3}}\right),
$$

where $\theta_{B}^{\prime}$ is the azimuthal angle of the vector $\mathbf{x}_{B S}^{\prime}$ in a spherical coordinate system centered at droplet $B$, and $\theta_{A}$ and $\phi_{A}$ are the azimuthal and meridional angles of the vector $\mathbf{x}_{A s}$ in a coordinate system centered at droplet $A$,

$$
\begin{aligned}
r= & \left(R_{A}^{2}+R_{B}^{2}+L^{2}-2 R_{A} R_{B} \cos \left(\theta^{*}\right)-2 R_{A} L \cos \left(\theta_{A}\right)\right. \\
& \left.-2 R_{B} L \cos \left(\theta_{B}^{\prime}\right)\right)^{1 / 2}
\end{aligned}
$$

is the distance between the position $\mathbf{x}_{A s}$ on droplet $A$ and $\mathbf{x}_{B s}^{\prime}$ on droplet $B$, and

$$
\cos \left(\theta^{*}\right)=-\cos \left(\theta_{A}\right) \cos \left(\theta_{B}^{\prime}\right)-\sin \left(\theta_{A}\right) \sin \left(\theta_{B}^{\prime}\right) \cos \left(\phi_{A}\right)
$$

is the cosine of the angle between the vectors $\mathbf{x}_{A s}$ and $\mathbf{x}_{B s}^{\prime}$. The expression (A7) is expanded in a Taylor series in the parameter $(1 / L)$

$$
n_{i}\left(\mathbf{x}_{A s}\right) J_{i j}\left(\mathbf{x}_{A s}-\mathbf{x}_{B s}^{\prime}\right) n_{j}\left(\mathbf{x}_{B s}^{\prime}\right)=\frac{l_{m}\left(\theta_{A}, \phi_{A}, \theta_{B}\right)}{L^{m}}
$$

and the coefficients $g_{m n}$ are evaluated from the coefficients $l_{m}$

$g_{m n}=\frac{(2 n+1)}{4 \pi R_{A}^{2}} \int d \mathbf{x}_{A s} l_{m}\left(\theta_{A}, \phi_{A}, \theta_{B}\right) P_{n}\left(\cos \left(\theta_{A}\right)\right)$

The following coefficients $g_{m n}$ for $(0 \leqslant m \leqslant 5)$ and $(0 \leqslant n$ $\leqslant 5)$ are not zero: 


$$
\begin{aligned}
g_{11}= & -2 \cos \left(\theta_{B}^{\prime}\right), \\
g_{21}= & R_{B}-3 \cos \left(\theta_{B}^{\prime}\right)^{2} R_{B}, \\
g_{22}= & -2 \cos \left(\theta_{B}^{\prime}\right) R_{A}, \\
g_{31}= & \left(2 \cos \left(\theta_{B}^{\prime}\right) R_{A}^{2}\right) / 5+4 \cos \left(\theta_{B}^{\prime}\right) R_{B}^{2}-6 \cos \left(\theta_{B}^{\prime}\right)^{3} R_{B}^{2}, \\
g_{32}= & 2 R_{A} R_{B}-6 \cos \left(\theta_{B}^{\prime}\right)^{2} R_{A} R_{B}, \\
g_{33}= & \left(-12 \cos \left(\theta_{B}^{\prime}\right) R_{A}^{2}\right) / 5, \\
g_{41}= & \left(-3 R_{A}^{2} R_{B}\right) / 5+\left(9 \cos \left(\theta_{B}^{\prime}\right)^{2} R_{A}^{2} R_{B}\right) / 5-\left(3 R_{B}^{3}\right) / 2 \\
& +12 \cos \left(\theta_{B}^{\prime}\right)^{2} R_{B}^{3}-\left(25 \cos \left(\theta_{B}^{\prime}\right)^{4} R_{B}^{3}\right) / 2, \\
g_{42}= & \left(6 \cos \left(\theta_{B}^{\prime}\right) R_{A}^{3}\right) / 7+12 \cos \left(\theta_{B}^{\prime}\right) R_{A} R_{B}^{2} \\
& -18 \cos \left(\theta_{B}^{\prime}\right)^{3} R_{A} R_{B}^{2}, \\
g_{43}= & \left(18 R_{A}^{2} R_{B}\right) / 5-\left(54 \cos \left(\theta_{B}^{\prime}\right)^{2} R_{A}^{2} R_{B}\right) / 5, \\
g_{44}= & \left(-20 \cos \left(\theta_{B}^{\prime}\right) R_{A}^{3}\right) / 7, \\
g_{51}= & \left(-18 \cos \left(\theta_{B}^{\prime}\right) R_{A}^{2} R_{B}^{2}\right) / 5+6 \cos \left(\theta_{B}^{\prime}\right)^{3} R_{A}^{2} R_{B}^{2} \\
& -\left(33 \cos \left(\theta_{B}^{\prime}\right) R_{B}^{4}\right) / 4, \\
& +\left(65 \cos \left(\theta_{B}^{\prime}\right)^{3} R_{B}^{4}\right) / 2-\left(105 \cos \left(\theta_{B}^{\prime}\right)^{5} R_{B}^{4}\right) / 4, \\
& -42 \cos \left(\theta_{B}^{\prime}\right)^{3} R_{A}^{2} R_{B}^{2}, \\
g_{52}= & \left(-12 R_{A}^{3} R_{B}\right) / 7+\left(36 \cos \left(\theta_{B}^{\prime}\right)^{2} R_{A}^{3} R_{B}\right) / 7-6 R_{A} R_{B}^{3} \\
& +48 \cos \left(\theta_{B}^{\prime}\right)^{2} R_{A} R_{B}^{3}-50 \cos \left(\theta_{B}^{\prime}\right)^{4} R_{A} R_{B}^{3}, \\
g_{53}= & \left(4 \cos \left(\theta_{B}^{\prime}\right) R_{A}^{4}\right) / 3+\left(138 \cos \left(\theta_{B}^{\prime}\right) R_{A}^{2} R_{B}^{2}\right) / 5 \\
g_{55}= & \left(-10 \cos \left(\theta_{B}^{\prime}\right) R_{A}^{4}\right) / 3 . \\
& \left(120 \cos \left(\theta_{B}^{\prime}\right)^{2} R_{A}^{3} R_{B}\right) / 7, \\
& \\
&
\end{aligned}
$$

Using the above relations, the results for $V_{A A}^{(n)}$ and $V_{A B}^{(n)}$ for $0 \leqslant n \leqslant 5$, correct to $O\left(1 / L^{5}\right)$, are

$$
\begin{aligned}
V_{A A}^{(0)}= & 0, \\
V_{A A}^{(1)}= & \frac{K}{\mu}\left[\frac{4 c_{A}^{*} c_{B}^{*} R_{B}}{5 L^{2}}-\frac{4}{5 L^{3}}\left(c_{A}^{* 2} R_{A} R_{B}+c_{B}^{* 2} R_{B}^{2}\right)\right. \\
& +\frac{12 c_{A}^{*} c_{B}^{*} R_{B}^{2} R_{A}}{5 L^{4}} \\
& \left.-\frac{8}{5 L^{5}}\left(\left(c_{A}^{* 2}+c_{B}^{* 2}\right) R_{B}^{2} R_{A}\left(R_{A}+R_{B}\right)\right)\right],
\end{aligned}
$$

$$
\begin{aligned}
V_{A A}^{(2)}= & \frac{K}{\mu}\left[\frac{4 c_{A}^{*} c_{B}^{*} R_{A} R_{B}}{L^{3}}-\frac{4 R_{A} R_{B}}{35 L^{4}}\left(5 c_{A}^{* 2} R_{A}+8 c_{B}^{* 2} R_{B}\right)\right. \\
& +\frac{12 c_{A}^{*} c_{B}^{*} R_{A}^{2} R_{B}^{2}}{5 L^{5}}, \\
V_{A A}^{(3)}= & \frac{K}{\mu}\left[\frac{8 c_{A}^{*} c_{B}^{*} R_{A}^{2} R_{B}}{15 L^{4}}-\frac{8 R_{A}^{2} R_{B}}{105 L^{5}}\left(7 c_{A}^{* 2} R_{A}+16 c_{B}^{* 2} R_{B}\right)\right], \\
V_{A A}^{(4)}= & \frac{K}{\mu} \frac{40 c_{A} c_{B} R_{A}^{3} R_{B}}{77 L^{5}}, \\
V_{A A}^{(5)}= & 0, \\
V_{A B}^{(0)}= & 0, \\
V_{A B}^{(1)}= & \frac{K}{\mu}\left[-\frac{c_{A}^{*} c_{B}^{*} R_{A} R_{B}}{L^{3}}+\frac{R_{A} R_{B}\left(c_{A}^{* 2} R_{A}+c_{B}^{* 2} R_{B}\right)}{L^{4}}\right. \\
& \left.-\frac{c_{A}^{*} c_{B}^{*} R_{A} R_{B}\left(20 R_{A} R_{B}-R_{A}^{2} R_{B}^{2}\right)}{5 L^{5}}\right], \\
V_{A B}^{(2)=}= & \frac{K}{\mu}\left[-\frac{c_{A}^{*} c_{B}^{*} R_{A}^{2} R_{B}}{L^{4}}+\frac{R_{A}^{2}}{L^{5}}\left(c_{A}^{* 2} R_{A} R_{B}-c_{B}^{* 2} R_{B}^{2}\right)\right], \\
V_{A B}^{(3)=} & \frac{K}{\mu}\left[-\frac{6 c_{A}^{*} c_{B}^{*} R_{A}^{3} R_{B}}{5 L^{5}}\right], \\
V_{A B}^{(4)}= & 0, \\
V_{A B}^{(5)}= & 0 .
\end{aligned}
$$

${ }^{1}$ E. D. Siggia, Phys. Rev. A 20, 595 (1979).

${ }^{2}$ M. San Miguel, M. Grant, and J. D. Gunton, Phys. Rev. A 31, 1001 (1985).

${ }^{3}$ H. Furukawa, Phys. Rev. A 31, 1103 (1985).

${ }^{4}$ K. Kawasaki and T. Ohta, Physica (Utrecht) 118A, 175 (1983).

${ }^{5}$ V. Kumaran, J. Chem. Phys. 109, 7644 (1998).

${ }^{6}$ V. Kumaran, J. Chem. Phys. 109, 3240 (1998).

${ }^{7}$ V. Kumaran, J. Chem. Phys. 109, 2437 (1998).

${ }^{8}$ G. Gonella, E. Orlandini, and J. M. Yeomans, Phys. Rev. Lett. 78, 1695 (1997).

${ }^{9}$ E. Orlandini, M. R. Swift, and J. M. Yeomans, Europhys. Lett. 32, 463 (1995).

${ }^{10}$ I. M. Lifshitz and V. V. Slyozov, J. Phys. Chem. Solids 19, 35 (1961).

${ }^{11} \mathrm{~J}$. D. Gunton and M. Droz, Introduction to the Theory of Metastable and Unstable States (Springer-Verlag, Berlin, 1983).

${ }^{12}$ H. Tanaka, J. Chem. Phys. 103, 2361 (1995).

${ }^{13}$ H. Tanaka, J. Chem. Phys. 105, 10099 (1996).

${ }^{14}$ N. C. Wong and C. M. Knobler, Phys. Rev. A 24, 3205 (1981).

${ }^{15}$ F. S. Bates and P. Wiltzius, J. Chem. Phys. 91, 3258 (1989).

${ }^{16}$ P. C. Hohenberg and B. I. Halperin, Rev. Mod. Phys. 49, 435 (1977).

${ }^{17}$ H. Tanaka, J. Chem. Phys. 107, 3734 (1997).

${ }^{18}$ N. Vladimirova, A. Malagoli, and R. Mauri, Phys. Rev. E 60, 2037 (1998).

${ }^{19}$ V. Kumaran and D. L. Koch, Phys. Fluids A 5, 1123 (1993). 